\title{
Therapeutic Effect of Cabazitaxel and Blood-Brain Barrier opening in a Patient-Derived Glioblastoma Model
}

\author{
Einar Sulheim ${ }^{1,2,3}$, Yrr Mørch², Sofie Snipstad 1,2,3, Sven Even Borgos², Hrvoje Miletic ${ }^{4,5}$, Rolf Bjerkvig5,6, \\ Catharina de Lange Davies ${ }^{1}$, Andreas K.O. Åslund1,2,7 \\ 1. Department of Physics, Norwegian University of Science and Technology (NTNU), Trondheim, Norway \\ 2. Department of Biotechnology and Nanomedicine, SINTEF AS, Trondheim Norway \\ 3. Cancer Clinic, St.Olav's University Hospital, Trondheim Norway \\ 4. Department of Pathology, Haukeland University Hospital, Bergen, Norway \\ 5. Department of Biomedicine, University of Bergen, Norway \\ 6. Department of Oncology, Luxembourg Institute of Health, Luxembourg \\ 7. Stroke Unit, Department of internal medicine, St. Olav's University Hospital, Trondheim, Norway \\ $\bowtie$ Corresponding author: einar.sulheim@ntnu.no \\ (c) Ivyspring International Publisher. This is an open access article distributed under the terms of the Creative Commons Attribution (CC BY-NC) license \\ (https://creativecommons.org/licenses/by-nc/4.0/). See http://ivyspring.com/terms for full terms and conditions.
}

Received: 2018.11.15; Accepted: 2019.02.05; Published: 2019.02.07

\begin{abstract}
Treatment of glioblastoma and other diseases in the brain is especially challenging due to the blood-brain barrier, which effectively protects the brain parenchyma. In this study we show for the first time that cabazitaxel, a semi-synthetic derivative of docetaxel can cross the blood-brain barrier and give a significant therapeutic effect in a patient-derived orthotopic model of glioblastoma. We show that the drug crosses the blood-brain barrier more effectively in the tumor than in the healthy brain due to reduced expression of $\mathrm{p}$-glycoprotein efflux pumps in the vasculature of the tumor. Surprisingly, neither ultrasound-mediated blood-brain barrier opening (sonopermeation) nor drug formulation in polymeric nanoparticles could increase either accumulation of the drug in the brain or therapeutic effect. This indicates that for hydrophobic drugs, sonopermeation of the blood brain barrier might not be sufficient to achieve improved drug delivery. Nonetheless, our study shows that cabazitaxel is a promising drug for the treatment of brain tumors.
\end{abstract}

Key words: cabazitaxel, glioblastoma, orthotopic model, sonopermeation

\section{Introduction}

Glioblastoma (GBM) represents one of the worst prognoses among cancers, with a median survival of 14.6 months [1]. GBM typically exhibits rapid, invasive growth, insensitivity to radiation and chemotherapy and often an intact blood brain barrier (BBB) $[2,3]$. The standard therapy for GBM consists of a combination of surgery, radiotherapy and temozolomide. Partly due to the intact BBB, the selection of effective drugs is highly limited. Temozolomide is a small lipophilic drug known to cross the BBB to some extent $[1,4]$, but methods to deliver this drug more efficiently across the BBB are sought [5].
Cabazitaxel (cab) (Jevtana ${ }^{\circledR}$, Sanofi-Aventis) is a highly potent taxane used as a second line treatment in docetaxel-resistant prostate cancer presumably due to its lower affinity to the efflux transporter P-glycoprotein (P-gp)[6, 7]. It has been evaluated without success in a phase II study for effect in temozolomide refractory glioma (clinicaltrials.gov NCT 01866449).

One of the most promising methods for drug delivery across the BBB is targeted sonopermeation [8-11] where focused ultrasound (FUS) and exogenous microbubbles (MBs) are used to enable permeation through the $\mathrm{BBB}$, presumably by disrupting the tight 
junctions between endothelial cells and thereby increasing extravasation of drugs [12], and/or downregulating P-gp [13-16]. The effects of sonopermeation are generally considered safe and transient, but a recent study has challenged this view [17]. Especially for GBM, which is often a localized disease, targeting by FUS is promising for increased local drug delivery, a strategy that is also currently under evaluation in clinical trials $[18,19]$.

While using FUS and MBs for BBB opening is quite well established, it is not clear how far into the brain parenchyma the method can deliver various forms of drugs. It has been hypothesized that sonopermeation can be especially promising in the combination with macromolecules and nanomedicines as these are unable to extravasate from intact vessels. Nanomedicines also have the potential advantages of reducing side effects, controlling drug delivery and allowing for delivery of pharmaceuticals such as hydrophobic drugs, proteins and nucleic acids[20].

We have previously presented an inhouse technology consisting of biodegradable poly (alkyl cyanoacrylate) (PACA) nanoparticles (NPs) that can be used to stabilize MBs [21, 22], and used them to deliver the NPs both to solid tumors [23] and across the BBB $[24,25]$. In the current study we aimed to treat mice with a patient derived xenograft (PDX)-model of GBM that closely resembles the human disease [26-28]. The model is invasive and independent of angiogenesis, suggesting that the BBB should be intact and present a barrier to drug delivery. We hypothesized that cab can be a useful drug in the treatment of GBM and investigated the effect of sonopermeation of the BBB on both the delivery of cab and cab-loaded NPs (cab-NPs).

\section{Materials and methods \\ NPs, NPMBs and Cab}

Empty or cab-loaded poly(2-ethyl butyl cyanoacrylate) (PEBCA) NPs (cab-NPs) and NP stabilized microbubbles (NPMBs) were synthesized as described previously [21, 23, 24]. Briefly, the NPs were made in a one-step mini emulsion polymerization where the water phase contained the surfactants Brij L23 (10 mM, 23 PEG units, MW 1225, Sigma- Aldrich) and Kolliphor HS15 (10 mM, 15 PEG units, MW 960, Sigma-Aldrich) in $0.1 \mathrm{M} \mathrm{HCl}$ and the oil phase 2-ethyl butyl cyanoacrylate, Miglyol 812 (co-stabilizer, Cremer) methane sulphonic acid (Sigma-Aldrich), azo bis-dimethyl valeronitrile (V65, oil-soluble radical initiator, Waco) and $10 \% \mathrm{w} / \mathrm{v}$ cab for the drug loaded NPs. The miniemulsion was formed by sonicating for 3 minutes on ice $(6 \times 30$ second intervals, $60 \%$ amplitude, Branson Ultrasonics digital sonifier 450). The NPs were dialyzed to remove unreacted surfactants. NPMBs were made by mixing $1 \% \mathrm{w} / \mathrm{v}$ NPs with $0.5 \% \mathrm{w} / \mathrm{v}$ Casein (Sigma) in $0.9 \%$ PBS using an Ultra Turrax (Branson Ultrasonics). The clinical formulation of cab was made following the protocol for Jevtana ${ }^{\circledR}$. Cab (TXD-258, Biochempartner Co. Ltd.,) was dissolved to $40 \mathrm{mg} / \mathrm{ml}$ in Tween- 80 (Sigma-Aldrich) and further dissolved 1:4 in 13\% ethanol.

Size and size distribution of the NPs and cab was measured by dynamic light scattering (DLS, Zetasizer Nano ZS, Malvern Instruments). Drug loading was measured by LC-MS/MS as described previously [23]. NPMBs were imaged in a Countess cell counting chamber (ThermoFisher) and analyzed for size, concentration and size distribution with ImageJ. NPs and NPMBs were imaged and visually inspected by scanning electron microscopy on either a SEM APREO (NPs) (FEI), or a S(T)EM (NPMBs) (Hitachi). The NPMBs were sputter coated with $5 \mathrm{~nm}$ gold prior to imaging.

\section{Cell culture}

The GBM cell line P3 was derived from a patient biopsy and has been characterized previously [29]. Cells were cultured in Neurobasal medium (Gibco), supplemented with B27 $(20 \mu \mathrm{l} / \mathrm{ml}$, Gibco $)$, GlutaMax-1 (10 $\mu \mathrm{l} / \mathrm{ml}$, Gibco), fibroblast growth factor-2 (FGF-2, $20 \mathrm{ng} / \mathrm{ml}$, Peprotech) and Heparin (64 IU/ml, LEO).

\section{In vitro toxicity}

For in vitro toxicity, cells were grown in 96well plates (10000 cells/well) for 4 days before cab-NPs, empty NPs or cab was added to the wells at concentrations ranging from $0.003 \mathrm{ng} / \mathrm{ml}$ to 200 $\mathrm{ng} / \mathrm{ml}$. Cells were incubated for 72 hours. Cytotoxicity was then measured by the AlamarBlue assay (Thermo Fisher Scientific). AlamarBlue was diluted 1:10 in cell medium and added to the wells. After 4 hours incubation, fluorescence at ex/em $550 / 590 \mathrm{~nm}$ was measured using a plate reader (SpectraMax i3, Molecular Devices).

\section{Animals and tumor inoculation}

Female Nod/SCID mice and Balb/c nude mice where purchased from Janvier Labs at 8 weeks of age and housed in specific pathogen free conditions at $22-23{ }^{\circ} \mathrm{C}, 50-60 \%$ relative humidity. The mice had free access to food and sterile water. All experimental procedures were approved by the Norwegian Animal Research Authorities.

Before inoculation of the glioma cells, the animals were anaesthetized using isoflurane $(\sim 2 \%$ in $78 \%$ medical air $\left./ 20 \% \mathrm{O}_{2}\right)$. Tamgesic $(1: 10,0.1 \mathrm{ml} / 20$ 
g) was given as local analgesic subcutaneously on the scalp. The skin was sterilized by ethanol and a $1 \mathrm{~cm}$ sagittal incision was made to expose the bregma. A hole was drilled at the coordinates $\mathrm{A}+1, \mathrm{~L}-2$ and $\mathrm{V}$ $-3.5 \mathrm{~mm}$ in relation to bregma. $5 \mu \mathrm{l}$ cell suspension (200 000 cells) was aspirated into a $25 \mu \mathrm{l}$ Hamilton syringe (Model $1702 \mathrm{~N}$ ). The syringe was mounted onto a stereotactic frame and inserted into the brain slowly for $4 \mathrm{~mm}$ and then retracted $0.5 \mathrm{~mm}$, before the injection. The injection was performed over 3 minutes and 2 minutes later the syringe was slowly retracted. The scalp was sutured and Marcain (1:5, $0.04 \mathrm{ml} / 20 \mathrm{~g})$ was injected subcutaneously on the scalp for long-term analgesia, while $2 \mathrm{~mL}$ saline was injected subcutaneously for hydration purposes during the recovery.

\section{Monitoring / MRI imaging}

Magnetic resonance imaging (MRI) was performed on a $7.05 \mathrm{~T}$ horizontal bore magnet (Biospec 70/20 Avance III, Bruker Biospin) with an 86 $\mathrm{mm}$ volume resonator for RF transmission and a phased array mouse brain surface coil for reception. From week three post inoculation, the animals were scanned on a weekly basis to evaluate tumor development using a T2-RARE weighted protocol. The animals were anesthetized using isoflurane ( $2 \%$ in $78 \%$ medical air $/ 20 \% \mathrm{O} 2$ ).

T2-RARE: TE/TR 54/2000 ms, RARE factor 16, zero fill acceleration of $1.3,14$ averages, lasting 5 minutes and 36 seconds. The geometry of the MR sequence had a field of view of $20 \mathrm{~mm} \times 20 \mathrm{~mm}$, matrix size of 200x200 and 9 slices á $1 \mathrm{~mm}$.

\section{Treatment}

Treatment was initiated after 5 weeks when the tumors had reached approximately $15 \mathrm{~mm}^{3}$. The animals were randomly divided into four groups and treated with 1: cab-NPMB and FUS $(\mathrm{N}=3), 2$ : cab, empty NPMB and FUS $(\mathrm{N}=4), 3$ : cab $(\mathrm{N}=4), 4$ : Saline control $(\mathrm{N}=4)$, as visualized in Figure 1 . The animals received two weekly treatments. In group 1 the cab-NPMBs were injected immediately before FUS, in group 2 free cab was injected followed by empty NPMBs, before FUS. In a separate set of animals the tumors were allowed to grow for one additional week to get tumors with sizes similar to the average in the treatment groups and the animals were euthanized 3 hours after treatment to quantify cab content in the brain (3 mice / group). In this part of the study three balb/c nude mice where included due to challenges with delivery of NOD/Scid mice. Tumors grew similarly in this mouse strain.

In group 1 and 2, MRI-guided FUS was used to open the BBB/blood-tumor barrier (BTB) in and around the tumor. MRI images for guiding was acquired as described above (similarly as for monitoring growth), but a larger phased array rat brain surface coil, placed on top of the animal, was used for reception as the animals were laying in a supine position. For T2-RARE, the same sequence was used, and Fast-Low Angle Shot (FLASH) was used to evaluate the BBB-opening by extravasation of the gadolinium (Gd)-contrast agent Omniscan (GE Healthcare) into the brain parenchyma.

FLASH: Flip angle of $40^{\circ}$, TE/TR 3.5/200 ms, zero fill acceleration of $1.3,12$ averages, lasting 2 minutes and 22 seconds. The geometry of the MR sequence had a field of view of $20 \mathrm{~mm} \times 20 \mathrm{~mm}$, matrix size of 200x200 and 9 slices á $1 \mathrm{~mm}$.

The ultrasound equipment was a RK300 (FUS Instruments) with a $1.1 \mathrm{MHz}$ transducer. Four sonication targets in a $2 \times 2$ matrix (distanced $1.5 \mathrm{~mm}$ apart), were selected on each animal to cover the tumor. The sonication lasted for 6 minutes with a pulse repetition frequency of $1 \mathrm{~Hz}$, pulse length of 10000 cycles and an estimated in situ pressure of 0.32 $\mathrm{MPa}$. The attenuation through the mouse skull was $15 \%$, measured in previous experiments [24]. After injection of cab $(50 \mu \mathrm{l}, 10 \mathrm{mg} / \mathrm{kg}$ diluted in saline) through the tail vein, MBs were injected via the tail vein as $3 \times 50 \mu \mathrm{l}$ bolus injections after 0,2 and 4 minutes. Post treatment, a bolus injection of $\mathrm{Gd}$ was injected to evaluate the BBB opening.

Following the first treatment, the animals were imaged for assessment of tumor size weekly for four weeks. Then the animals in the control group had to be euthanized due to the tumor size, and animals in all groups were euthanized.

\section{Mass spectrometry (MS)-analysis of cab accumulation}

To quantify delivery of cab to the healthy brain and tumor, 3 hours after the treatment the mice were killed and the brains removed. The brains were divided in two (healthy and tumor-bearing hemisphere) and weighed. The two hemispheres were manually cut into pieces and digested with an enzyme buffer. The enzyme buffer consisted of Dulbecco's Modified Eagle Medium (DMEM, Thermo Fisher Scientific) with $1 \% \quad(\mathrm{v} / \mathrm{v})$ penicillin-streptomycin (Sigma-Aldrich) giving a final concentration of $100 \mathrm{U} / \mathrm{ml}$ penicillin and $100 \mu \mathrm{g} / \mathrm{ml}$ streptomycin, $0.125 \mathrm{mg} / \mathrm{ml}$ papain (Merck), 2.5 $\mathrm{mg} / \mathrm{ml}$ trypsin (Sigma-Aldrich), $0.8 \mathrm{mg} / \mathrm{ml}$ collagenase (Sigma-Aldrich), $0.69 \mathrm{mg} / \mathrm{ml}$ hyaluronidase (Sigma-Aldrich) and 1\% (v/v) Triton X-100 (Sigma-Aldrich). Freshly prepared enzyme buffer was added at $1 \mathrm{ml}$ per $50 \mathrm{mg}$ tissue. The samples were heated to $37^{\circ} \mathrm{C}$ for 72 hours with 
vortexing once a day, until the tissue was completely dissolved. The tissue digests were diluted $10 \mathrm{x}$ in acetone before centrifugation; this has the dual effect of both precipitating proteins and other macromolecules, thus cleaning up the sample, and making sure all cab is solubilized. Internal standard (hexadeuterated cab) dissolved in acetone was added to a final concentration of $10 \mathrm{ng} / \mathrm{ml}$ during the acetone dilution to correct for possible matrix effects.

Cab was quantified by LC-MS/MS, using an Agilent 1290 HPLC system coupled to an Agilent 6490 triple quadrupole MS. The HPLC column was an Ascentis Express C8, 75x2.1 mm, $2.7 \mu \mathrm{m}$ particle size with a $5 \times 2.1 \mathrm{~mm}$ guard column of the same material (Sigma-Aldrich), run at $40^{\circ} \mathrm{C}$. Eluent A was $25 \mathrm{mM}$ formic acid in water and eluent B was $100 \%$ methanol, and flow rate was $0.5 \mathrm{ml} /$ minute. The mobile phase gradient was isocratic at $55 \%$ eluent $B$ for 1.5 minute, then from $55 \%$ to $80 \%$ B over $1 \mathrm{~min}$, followed by 1 minute washout time and subsequently column re-equilibration. Injection volume was $5.0 \mu \mathrm{l}$. MS detection was in positive ESI mode (Agilent Jetstream) quantified in multiple reaction monitoring (MRM) mode using the transition $\mathrm{m} / \mathrm{z} 858.3 \rightarrow 577.2$. The parent ion was chosen to be the $\mathrm{Na}$ adduct as this gave the best sensitivity. Similarly, the hexadeuterated internal standard was detected on the $864.4 \rightarrow 583.2$ transition. Analytes were run at $380 \mathrm{~V}$ fragmentor and $20 \mathrm{~V}$ collision energy.

Reference standards were used for accurate quantification. The unlabeled cab standard was the same as used for synthesis. Hexadeuterated cab internal standard was purchased from Toronto Research Chemicals (99.6\% isotopic purity). Standards were dissolved in acetone and were used to build an unlabeled standard series spanning at least five concentration points.

\section{Histology and P-gp staining}

28 days after first treatment all animals were euthanized and the brains were embedded in formalin and H\&E-stained for histopathological assessment. In addition, the sections were stained for P-gp efflux pump expression using an anti-P-gp antibody (EPR10364-57, Abcam, 1:200 dilution) followed by incubation with HRP (horseradish peroxidase) Rabbit EnVision-Polymer and DAB+ (3,3'-Diaminobenzidine) Chromogen (both from DAKO). Stitched images of entire brain sections were acquired on a Zeiss LSM 800 at 20x magnification. Images were acquired in bright field mode using built-in functions for color correction and uneven illumination correction. For analysis of P-gp expression, 8 region of interests (ROIs) of $840 \times 1120$ pixels were randomly chosen from each of the areas: 1, Healthy: The hemisphere without tumor. 2, Tumor proximity: Tumor-bearing hemisphere but outside the tumor. 3, Tumor periphery: Areas in the tumor border containing a mix of healthy and tumor cells. 4, Tumor core: Areas within the tumor core. The areas were then analyzed for cell density and P-gp expression using the InForm ${ }^{\circledR}$ software (Perkin Elmer) by supervised training of the software to recognize nuclei and DAB-stained P-gp.

\section{Data Analysis}

Data analysis and statistics were performed in Graphpad Prism7®. Throughout the figures error bars show standard deviation and significance is denoted as ${ }^{*}=p<0.05,{ }^{* *}=p<0.01,{ }^{* * *}=p<0.001$ and $* * * *=p<0.0001$.

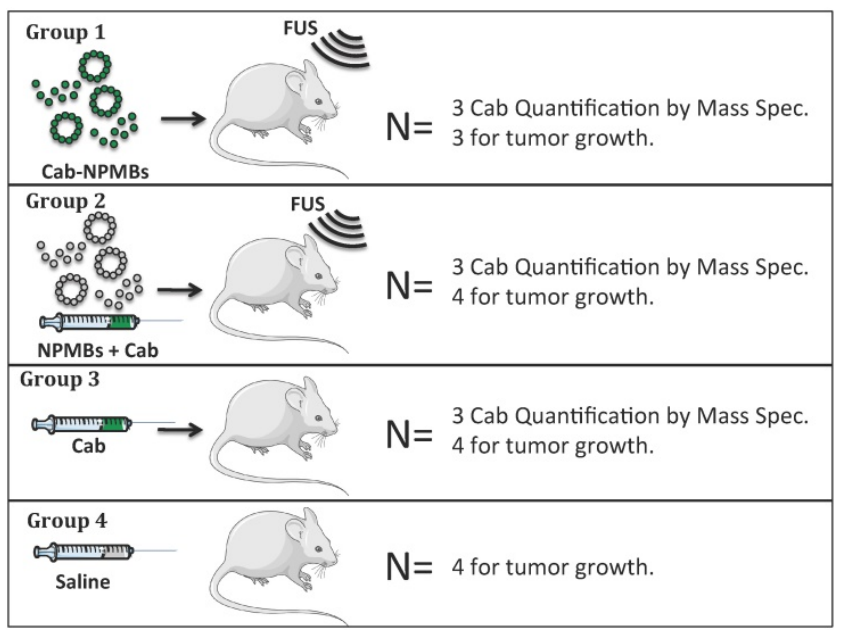

Figure 1: Studied treatment groups.

\section{Results}

Cab was formulated either as Jevtana, i.e. dissolved in Tween 80 and ethanol, or in PEBCA NPs. The cab-NPs had a diameter of $153 \mathrm{~nm}$, polydispersity index (PDI) of 0.19 , zetapotential of $-1.8 \mathrm{mV}$ and a cab loading capacity of $8.9 \% \mathrm{w} / \mathrm{w}$ (Figure 2). The empty NPs had a diameter of $162 \mathrm{~nm}$, PDI of 0.15 and zetapotential of $-2 \mathrm{mV}$. Jevtana forms micelles/clusters in aqueous solutions with a size around 10nm (Figure 2). The two formulations had similar effect on glioma cells in vitro with IC50 values around $20 \mathrm{ng} / \mathrm{ml}$ (Figure 2B) which is approximately 1000 times lower than empty NPs [30]. 


\section{A}
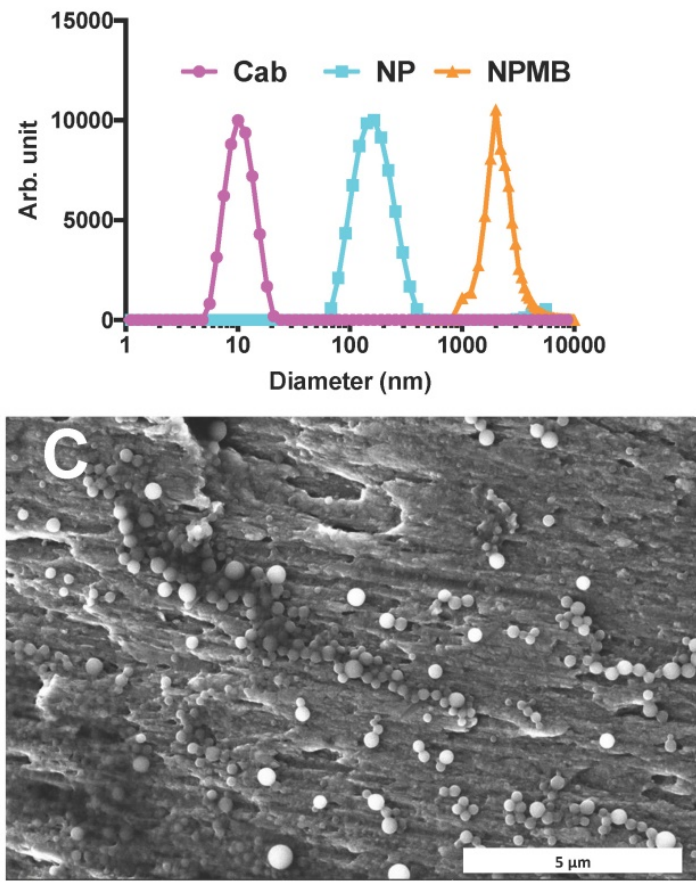

B
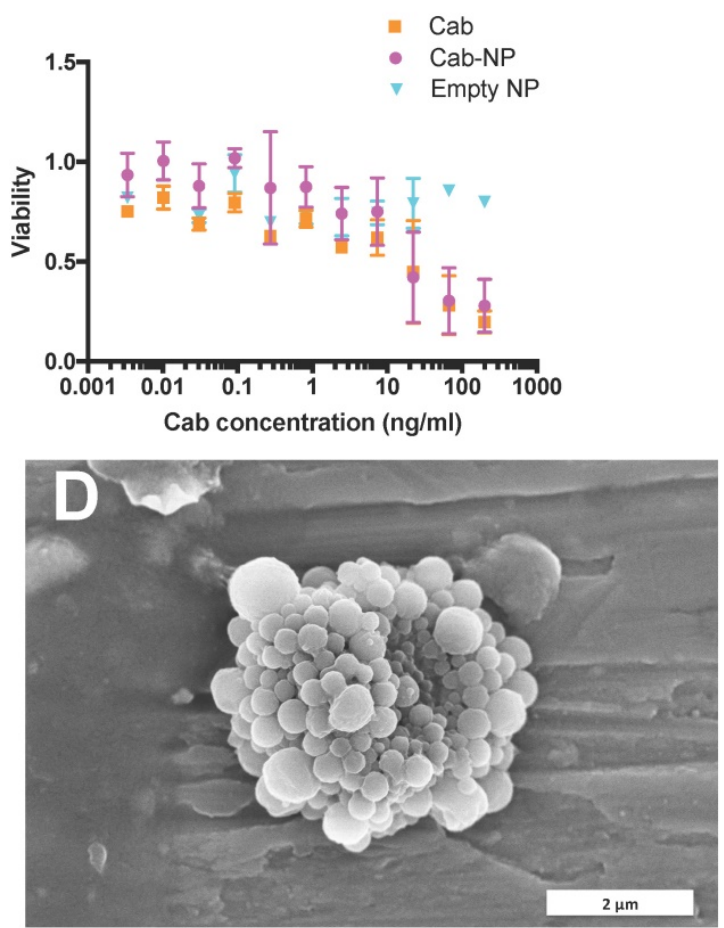

Figure 2: Characterization of the NP and MB platform. A: Diameter of cab, cab-NPs and cab-NPMBs by DLS or microscopy. B: Effect of cab, cab-NPs and empty NPs on patient derived glioblastoma cells in vitro $(n=2)$, error bars represent standard deviation. C: SEM image of the cab-NPs and D; SEM image of the empty NPMBs.

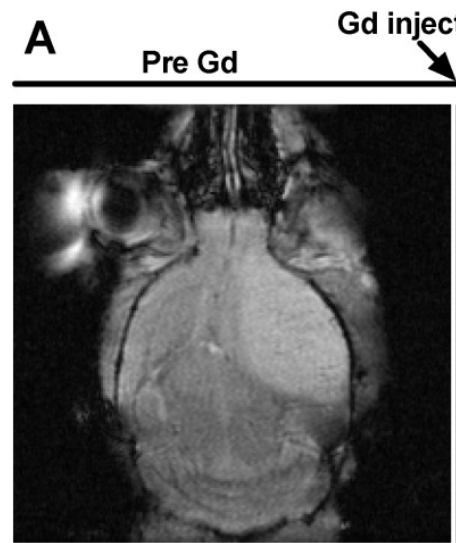

B

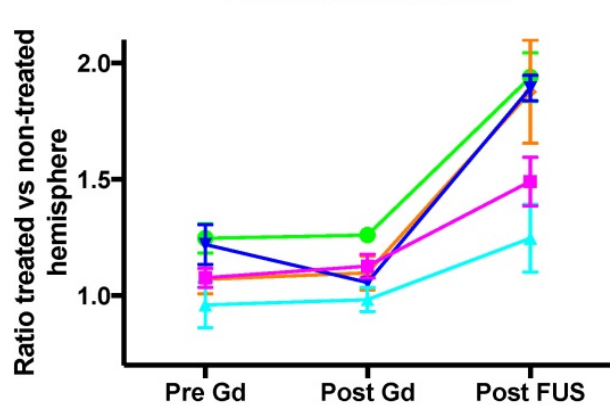

FUS treatment Gdinjection Post Gd Post Fus
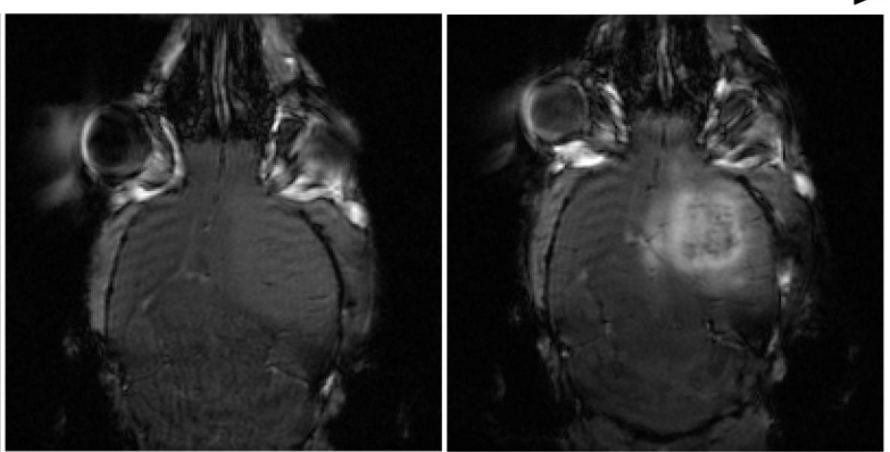

C

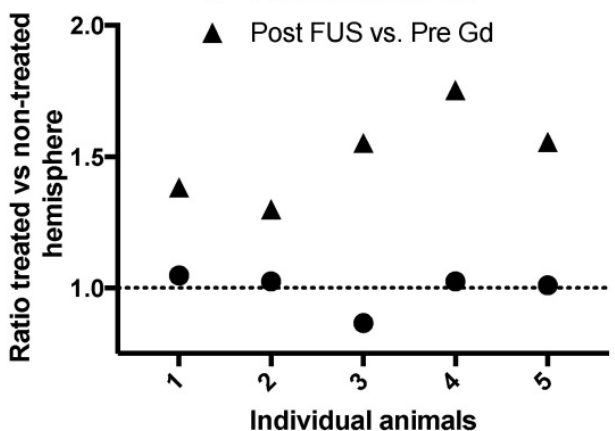

Figure 3: FUS mediated BBB-opening. A: FLASH images of the mouse brain before Gd injection, after Gd injection and after FUS treatment and Gd injection. B: Opening of BBB and extravasation of Gd. Signal from the tumor was compared to a mirrored ROI in the non-treated hemisphere, each line shows one animal, error bars show standard deviation of signal increase from three different sections of the tumor. The opening was measured in 5 animals. C: Variation between mice in BBB opening as measured by Gd extravasation. Pre Gd: Before injection of Gd. Post Gd: After injection of Gd, but before FUS treatment. Post FUS: After FUS treatment and with a new bolus injection of Gd. 
To investigate the leakiness of the BBB/BTB and the effect of FUS, we injected Gd contrast agent both before and after FUS and cab treatment. If the BBB or BTB was leaky, MRI would reveal a Gd-signal increase before FUS exposure. We observed no leakiness either in the healthy brain or in the tumor (Figure 3). However, after sonication and BBB opening, considerable signal increase could be observed in the sonicated region in all treated animals (Figure 3).

Following the observation that the BBB was intact also in the tumor, and not leaky to the MRI contrast agent, we aimed to investigate whether $\mathrm{cab}$ could cross the BBB and delay tumor progression. We also aimed to test if delivery of cab could be increased by formulation in NPs and permeating the BBB using FUS. The mice were injected intravenously with $c a b$ formulated as Jevtana or PACA NPMBs, and the BBB was opened using FUS and NPMBs (groups visualized in Figure 1.) A subgroup of animals was euthanized after 3 hours and the extracts from the brains were analyzed with LC-MS/MS. Interestingly, it was found that cab, regardless of delivery method (cab +/- FUS or cab-NPMBs + FUS) accumulated more in the hemisphere with the implanted tumor compared to the healthy hemisphere (Figure 4). A strong, positive correlation between amount of cab in tumor tissue and healthy tissue was observed (Figure 4B), and the tumor tissue had approximately 4 times more cab than healthy tissue. The correlation indicates that properties of the individual mice are decisive for the delivery of cab to the tumor (Figure 4B). There was a weak indication of less cab in the sonicated tumors (Figure 4A), indicating that BBB-opening could increase reflux of the drug back to the blood stream.

After finding that cab crossed the BTB to a larger extent than the $\mathrm{BBB}$, the therapeutic potential of the drug was evaluated by treating NOD/SCID mice with

the implanted PDX-glioma. Such experiments would reveal whether BBB opening, although not able to increase the accumulation/retention of the drug in the brain, could give improved therapeutic effect due to other mechanisms such as increased penetration into the brain parenchyma. All three treatment groups (cab-NP + FUS, cab + FUS, cab) had a significant therapeutic effect compared to the control group, but there was no difference between the treated groups (Figure 5). The treatment delayed the tumor growth significantly with the tumors reaching only a third of the size of the control tumors 3 weeks after the last treatment, i.e. all treated groups had an average size of approximately $70 \mathrm{~mm}^{3}$ compared to $250 \mathrm{~mm}^{3}$ in the control group (Figure 5).

At the endpoint of the treatment study, brains were sectioned and the tumor histology was evaluated and used to assess cell density and P-gp expression.

Since efflux pumps in the brain blood vessels are known to be an important barrier for delivery of many molecular drugs that would otherwise pass through the endothelial cells, and the tumor model was found to have a non-leaky phenotype when imaged by contrast enhanced MR imaging (Figure 3), we stained for P-gp in the brain sections (Figure 6). It was found that the level of P-gp-expression was down-regulated in the tumor blood vessels compared to healthy vessels, and the tumor cells did not express detectable levels of the efflux pump. Moreover, a slight trend suggesting a correlation between P-gp-expression in the tumors and treatment outcome was observed, but the correlation was not significant (Figure 6C). Far higher cellular density was measured in the tumor compared to the healthy brain (Figure 6D), but no indication of correlation between cellular density and treatment outcome was found (Figure $6 \mathrm{E})$. There was no significant change in P-gp expression in FUS treated animals (Figure

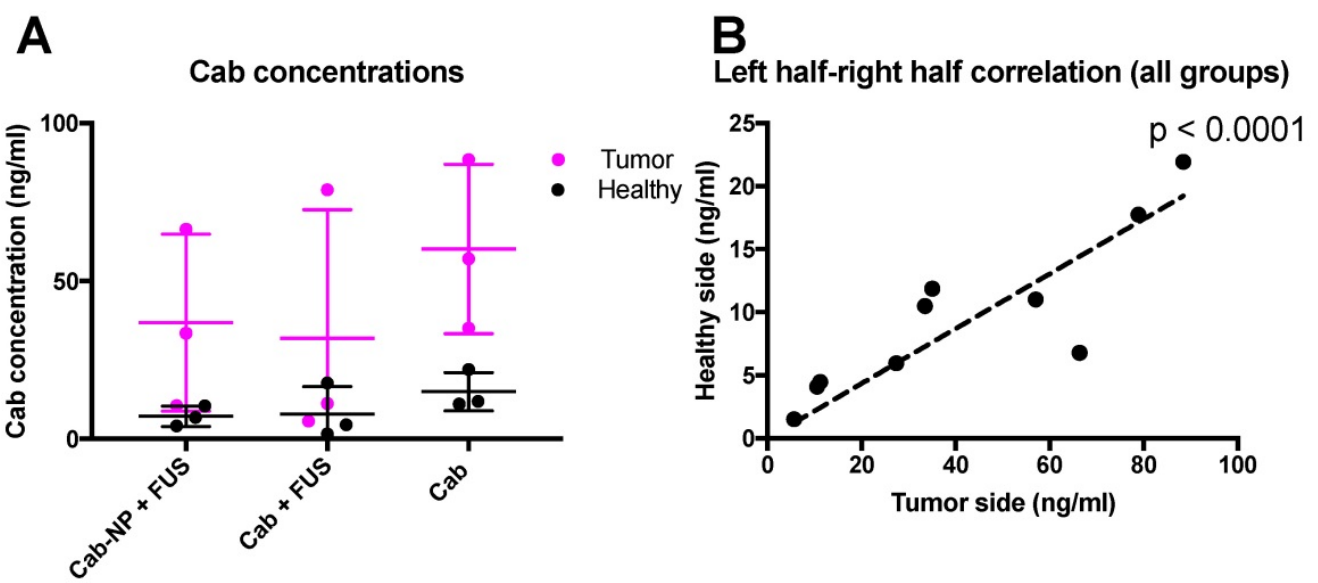

Figure 4: LC-MS/MS-analysis of cab in the brain and tumor of treated animals. A) Accumulation of cab in the tumor and healthy sides of the brain, mean of 3 animals in each group, error bars show standard deviation. B) Correlation between cab accumulation for all groups in the healthy and tumor-implanted hemisphere for the same 9 animals.
6F). In compliance with the correlation between $\mathrm{cab}$ in the right and left hemisphere (Figure $4 \mathrm{~B})$, and the possible connection between P-gp expression and tumor size (Figure 6C), there was a significant correlation between P-gp expression in the healthy hemisphere and the tumor (Figure $6 G)$. 


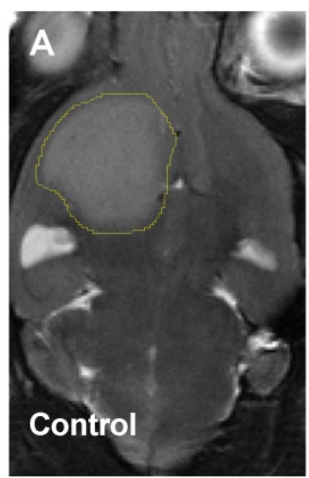

C

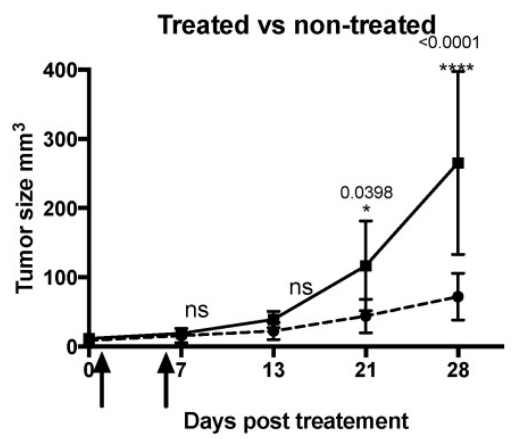

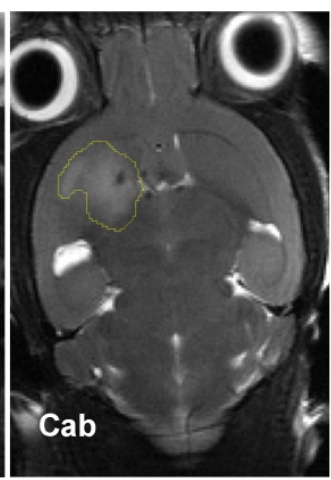
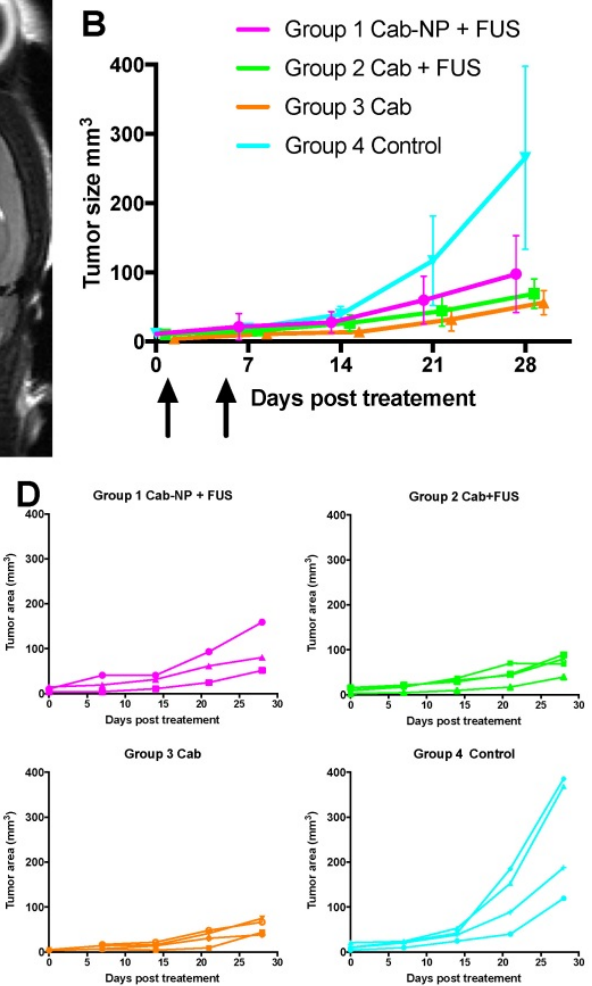

Figure 5: Therapeutic effect of cab. A: Representative tumor sizes at the end of the study. B: Average growth curves with standard deviation ( $N=3-4$ per group). Arrows show treatments. C: Statistical comparison of all the treated animals compared to the non-treated controls. D: Individual growth curves.
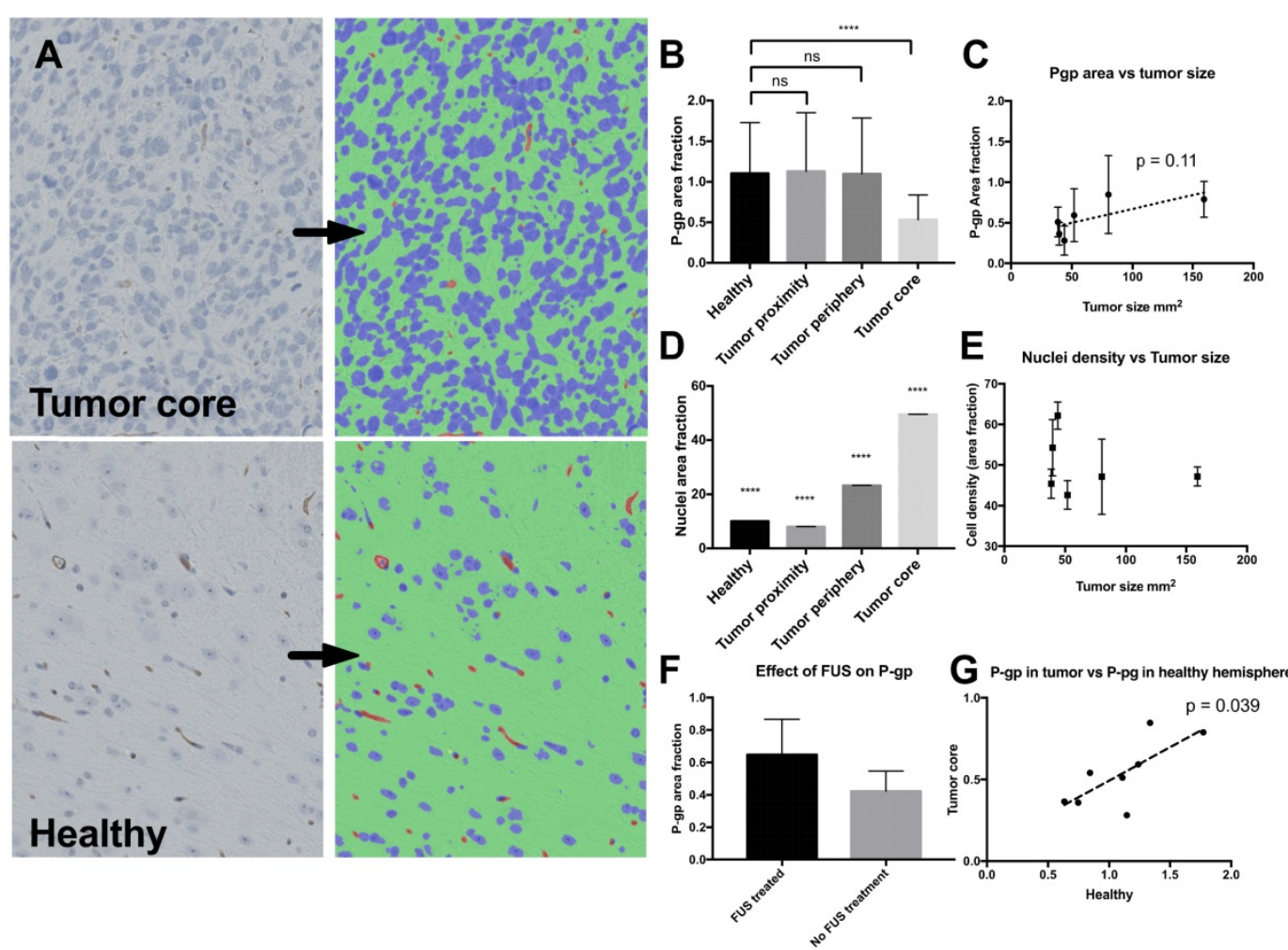

Figure 6: Expression of P-pg and cell density in the brain and tumor. A: Histology of the tumor (upper panel) and healthy area in the opposite hemisphere (lower panel). Bright field microscopy image (left) and result after automated segmentation into nuclei (blue), P-gp (brown) and other (green) B: P-gp expression in the healthy part of the brain and in the areas close to or in the tumor after automatic segmentation. C: Correlation of P-gp level in the tumor compared to tumor size at study completion. Each point show mean from one animal and error bars show standard deviation from 8 ROl's in the tumor. D: Area fraction of nuclei in the different areas, highest p-value compared to all other groups is shown. E: Area fraction of nuclei plotted against the final size of the tumor upon study completion. Each point shows one mean from 8 ROl's in the tumor and one animal. F: P-gp expression in sonicated and non-sonicated tumors, $n=4$ in each group. G: Correlation between P-gp expression in the healthy hemisphere and P-gp expression in the tumor. 


\section{Discussion}

Cab is a novel taxane that is reported to cross the BBB and has been suggested as a candidate for medication of primary tumors or metastases to the brain [31-33]. It has been shown to exhibit effect against in vitro glioma models [34] and in patient derived pediatric brain tumors [35], but to our knowledge never before tested in PDX-models of GBM. The drug is not in clinical use for treatment of brain tumors, but is approved as a second line treatment to docetaxel for castration resistant prostate cancer [36]. The use is generally hampered by severe adverse effects (primarily neutropenia) [37], but it has been demonstrated with multiple different cytostatic drugs that formulation in nanomedicines can ameliorate this toxicity [20]. However, encapsulation in NPs will change the biodistribution, and potentially impede crossing of the BBB. We have previously shown that the NPs used in this study do not significantly cross the intact BBB [24]. In the present study we report that the drug formulated either as used in the clinic today, or combined with FUS and MBs or in polymeric NPMB combined with FUS delay the growth of PDX- glioma to the same extent. Consistent with this, the MS measurement revealed that there was no significant difference in the accumulation of cab in the gliomas. Interestingly, the tumors did not show enhancement in contrast enhanced MRI prior to FUS treatment showing that the BBB was intact prior to FUS treatment and in the control group receiving cab only.

The efficient delivery of cab indicates that the drug is not effluxed back to the blood by P-gp. Cab is a substrate of P-gp, although at lower affinity than the other taxanes [38] and it is only able to cross the BBB to a limited degree and after saturation of the P-gp pumps [32]. The low amount of P-gp on endothelial cells in the tumor compared to healthy blood vessels, can explain the promising therapeutic response of cab. Consistent with this, we found indications that higher P-gp expression resulted in lower therapeutic effect and individual differences in P-gp expression in both BBB and BTB is the most likely explanation for the high correlation between cab accumulation in the healthy and tumor hemisphere. Why these genetically similar animals differ in P-gp expression is not known.

In order to compare the potential therapeutic effect of cab both in free and NP form, we compared the two after opening the BTB with FUS in the presence of MBs. FUS in combination with MBs is reported in several papers to create gaps between the endothelial cells or in the cell membrane, and allow for transport into the brain interstitium $[8,39]$. The role of P-gp in the efflux of the drug from the brain after BBB opening is not clear and could in principle be of limited importance once the NP/drug is deposited in the brain parenchyma and out of reach for the endothelial cells. However, this depends on how far from the blood vessels the drug travels after $\mathrm{BBB}$ opening. It is surprising that although sonopermeation of the BBB was confirmed by Gd-signal increase due to extravasation of the MRI-contrast agent, the FUS treatment did not increase accumulation of cab in any formulation. Hence, the differences between the formulations of cab and Gd, e.g. size, hydrophobicity and affinity for P-gp, means that Gd extravasation cannot be used to predict treatment efficacy, as is often assumed. In a similar study, Kobus et al. reported that only a subgroup of the treated animals had therapeutic benefit of BBB-opening [40]. In our histology sections we did not observe any down-regulation of P-gp in the FUS treated regions as observed by others [13-16]. However, the animals were killed 4 weeks after the last treatment and the stained sections can therefore not disclose anything on temporary effects short time after FUS treatment.

The accumulation of $\mathrm{cab}$ in the tumor hemisphere was around four times higher than in the healthy hemisphere in all treatment groups. This means that cab in its clinical formulation, as well as when combined with FUS and MBs, either free or encapsulated, crosses the BTB at least 4 times more efficiently (since the tumor does not cover the whole hemisphere) than the BBB. While there were rather large individual differences in cab accumulation between the animals, there was a striking correlation between magnitude of cab accumulation in the healthy hemisphere, and the hemisphere with the tumor implanted. This suggests that other factors than disease progression and BTB status have effects on the delivery of cab (and probably multiple other drugs) to the brain and P-gp expression might be such a factor.

The PDX disease model used was chosen due to its invasive phenotype and independecy of angiogenesis [26], suggesting an intact BTB. The integrity of BTB before and after FUS was verified by a bolus injection of $\mathrm{Gd}$, and no indications of leaky BTB was found before FUS treatment. As we have also seen in previous experiments, the BBB could be opened with FUS allowing for accumulation of Gd in the sonicated region [25]. This shows that the tumor model exhibited a mainly intact BTB and the effect of FUS with respect to Gd extravasation was similar to what we have observed for the $\mathrm{BBB}$ in healthy animals. Our study shows that successful opening of the BTB is not required to achieve a therapeutic effect of the drug alone. However, there are several studies 
demonstrating that drug delivery to the brain and brain tumors is hampered by the BBB/BTB and that opening the barrier with sonopermeation will increase the delivery [12, 41]. Our results show that all treatment groups performed similarly, but better than the control, and indicate that this is a field where more fundamental research is necessary to understand the underlying processes and effects. Our work indicates that opening of the BBB/BTB is not sufficient for improving the therapeutic response, but also the efflux pumps represent a major barrier for the drug.

Another important implication from this study is that invasive tumors, even low or non-angiogenic, can change the properties of the surrounding vasculature significantly as seen by reduction of P-gp expression. As our study is performed in a model from a single patient we cannot conclude on the generality of this observation. Future work should investigate if this occurs also in other GBM models and in patients with GBM.

While the lack of therapeutic gains from BBB/BTB opening by sonopermeation is surprising, our study shows that cab had a significant effect on the therapeutic response for this tumor model and is a drug that should be evaluated and considered in more detail for medication of brain tumors. We did not find increased therapeutic benefit from encapsulation in NPs, but nano-encapsulation could reduce the toxic effects of cab and be a useful alternative to the clinical formulation. Although our study lacks the comparison to standard of care and can therefore not be used to compare the effect of various treatments, it should be highlighted that invasive brain tumors are among the hardest to treat and any drug that can significantly slow the growth of these tumors are interesting candidates.

\section{Conclusion}

We have shown that non-angiogenic tumors can alter the expression of P-gp in the BTB and that this alteration is significant for accumulation of cab in the brain. Furthermore, we have shown that cab can reduce growth of a GBM PDX-tumor model, and this works for both free drug without FUS induced BTB-opening and for NPs with FUS induced BTB-opening. That NPs can be used to treat tumors in the brain with the same efficacy as normally formulated drug could be used to reduce the dose limiting side-effects often seen from cab.

\section{Acknowledgement}

Federica de Lorenzi at ExMi RWTH Aachen is thanked for help in histology analysis and training of the InForm segmentation system, Ida Sofie Jorstad and Ida Marie Høiaas for SEM imaging. SEM imaging was performed at the NTNU Nanolab and The Research Council of Norway is acknowledged for the support to the Norwegian Micro- and Nano-Fabrication Facility, NorFab, project number 245963/F50. Formulation/synthesis of NP, NPMB and cab, as well as tissue extraction and LC-MS/MS quantification of cab was performed at SINTEF, and Anne Rein Hatletveit and Catherine Løvmo are especially thanked for technical help. HES staining was performed by Ingunn Nervik at CMIC NTNU, MRI at the MR core facility NTNU, tumor implantation and animal housing at CoMed NTNU. The project was supported by The Central Norway Regional Health Authority, project number 46084000 and The Research Council of Norway project number 240316.

\section{Competing Interests}

The authors have declared that no competing interest exists.

\section{References}

1. Stupp R, Mason WP, van den Bent MJ, Weller M, Fisher B, Taphoorn MJB, et al. Radiotherapy plus concomitant and adjuvant temozolomide for glioblastoma. New Engl J Med. 2005; 352: 987-96.

2. Alves TR, Lima FRS, Kahn SA, Lobo D, Dubois LGF, Soletti R, et al. Glioblastoma cells: A heterogeneous and fatal tumor interacting with the parenchyma. Life Sci. 2011; 89: 532-9.

3. Sarkaria JN, Hu LS, Parney IF, Pafundi DH, Brinkmann DH, Laack NN, et al. Is the blood-brain barrier really disrupted in all glioblastomas? A critical assessment of existing clinical data. Neuro-Oncology. 2018; 20: 184-91.

4. Agarwala SS, Kirkwood JM. Temozolomide, a novel alkylating agent with activity in the central nervous system, may improve the treatment of advanced metastatic melanoma. Oncologist. 2000; 5: 144-51.

5. Wei KC, Chu PC, Wang HYJ, Huang CY, Chen PY, Tsai HC, et al. Focused Ultrasound-Induced Blood-Brain Barrier Opening to Enhance Temozolomide Delivery for Glioblastoma Treatment: A Preclinical Study. Plos One. 2013; 8.

6. Galsky MD, Dritselis A, Kirkpatrick P, Oh WK. Cabazitaxel. Nat Rev Drug Discov. 2010; 9: 677-8.

7. Vrignaud P, Semiond D, Lejeune P, Bouchard H, Calvet L, Combeau C, et al. Preclinical Antitumor Activity of Cabazitaxel, a Semisynthetic Taxane Active in Taxane-Resistant Tumors. Clin Cancer Res. 2013; 19: 2973-83.

8. Snipstad S, Sulheim E, de Lange Davies C, Moonen C, Storm G, Kiessling F, et al. Sonopermeation to improve drug delivery to tumors: from fundamental understanding to clinical translation. Expert Opin Drug Deliv. 2018: 1-13.

9. Choi JJ, Wang SG, Tung YS, Morrison B, Konofagou EE. Molecules of Various Pharmacologically-Relevant Sizes Can Cross the Ultrasound-Induced Blood-Brain Barrier Opening in Vivo. Ultrasound Med Biol. 2010; 36: 58-67.

10. Hynynen $\mathrm{K}$, McDannold N, Vykhodtseva N, Jolesz FA. Noninvasive MR imaging-guided focal opening of the blood-brain barrier in rabbits. Radiology. 2001; 220: 640-6.

11. Burgess A, Hynynen K. Drug delivery across the blood-brain barrier using focused ultrasound. Expert Opin Drug Del. 2014; 11: 711-21.

12. Sheikov N, McDannold N, Sharma S, Hynynen K. Effect of focused ultrasound applied with an ultrasound contrast agent on the tight junctional integrity of the brain microvascular endothelium. Ultrasound Med Biol. 2008; 34: 1093-104.

13. Aryal M, Fischer K, Gentile C, Gitto S, Zhang YZ, McDannold N. Effects on P-Glycoprotein Expression after Blood-Brain Barrier Disruption Using Focused Ultrasound and Microbubbles. Plos One. 2017; 12.

14. Zhang Z, Xu K, Bi YH, Yu GB, Wang SW, Qi X, et al. Low Intensity Ultrasound Promotes the Sensitivity of Rat Brain Glioma to Doxorubicin by Down-Regulating the Expressions of P-Glucoprotein and Multidrug Resistance Protein 1 In Vitro and In Vivo. Plos One. 2013; 8

15. Wan CPL, Jackson JK, Pirmoradi F, Chiao M, Burt HM. Increased Accumulation and Retention of Micellar Paclitaxel in Drug-Sensitive and P-Glycoprotein-Expressing Cell Lines Following Ultrasound Exposure. Ultrasound Med Biol. 2012; 38: 736-44.

16. Cho H, Lee HY, Han M, Choi JR, Ahn S, Lee T, et al. Localized Down-regulation of P-glycoprotein by Focused Ultrasound and Microbubbles induced Blood-Brain Barrier Disruption in Rat Brain. Sci Rep-Uk. 2016; 6. 
17. Kovacs ZI, Kim S, Jikaria N, Qureshi F, Milo B, Lewis BK, et al. Disrupting the blood-brain barrier by focused ultrasound induces sterile inflammation. Proc Natl Acad Sci U S A. 2017; 114: E75-E84

18. Lipsman N, Ironside S, Alkins R, Bethune A, Huang YX, Perry J, et al. Initial experience of blood-brain barrier opening for chemotherapeutic-drug delivery to brain tumours by MR-guided focused ultrasound. Neuro-Oncology. 2017; 19: vi275.

19. Carpentier A, Canney M, Vignot A, Reina V, Beccaria K, Horodyckid C, et al. Clinical trial of blood-brain barrier disruption by pulsed ultrasound. Sci Transl Med. 2016; 8.

20. Shi JJ, Kantoff PW, Wooster R, Farokhzad OC. Cancer nanomedicine: progress, challenges and opportunities. Nat Rev Cancer. 2017; 17: 20-37.

21. Mørch Y, Hansen R, Berg S, Åslund AKO, Glomm WR, Eggen S, et al. Nanoparticle-Stabilized Microbubbles for Multimodal Imaging and Drug Delivery. Contrast Media and Molecular imaging. 2015.

22. Sulheim E, Baghirov H, von Haartman E, Boe A, Aslund AK, Morch Y, et al. Cellular uptake and intracellular degradation of poly(alkyl cyanoacrylate) nanoparticles. J Nanobiotechnology. 2016; 14: 1.

23. Snipstad S, Berg S, Morch Y, Bjorkoy A, Sulheim E, Hansen R, et al. Ultrasound Improves the Delivery and Therapeutic Effect of Nanoparticle-Stabilized Microbubbles in Breast Cancer Xenografts. Ultrasound Med Biol. 2017; 43: 2651-69.

24. Baghirov H, Snipstad S, Sulheim E, Berg S, Hansen R, Thorsen F, et al. Ultrasound-mediated delivery and distribution of polymeric nanoparticles in the normal brain parenchyma of a metastatic brain tumour model. Plos One. $2018 ; 13$.

25. Aslund AK, Berg S, Hak S, Morch Y, Torp SH, Sandvig A, et al. Nanoparticle delivery to the brain--By focused ultrasound and self-assembled nanoparticle-stabilized microbubbles. J Control Release. 2015; 220: 287-94.

26. Sakariassen PO, Prestegarden L, Wang J, Skaftnesmo KO, Mahesparan R, Molthoff $\mathrm{C}$, et al. Angiogenesis-independent tumor growth mediated by stem-like cancer cells. P Natl Acad Sci USA. 2006; 103: 16466-71.

27. Talasila KM, Soentgerath A, Euskirchen P, Rosland GV, Wang J, Huszthy PC, et al. EGFR wild-type amplification and activation promote invasion and development of glioblastoma independent of angiogenesis. Acta Neuropathol. 2013; 125: 683-98.

28. Wang J, Miletic H, Sakariassen PO, Huszthy PC, Jacobsen H, Brekka N, et al. A reproducible brain tumour model established from human glioblastoma biopsies. Bmc Cancer. 2009; 9 .

29. Keunen O, Johansson M, Oudin A, Sanzey M, Rahim SAA, Fack F, et al. Anti-VEGF treatment reduces blood supply and increases tumor cell invasion in glioblastoma. P Natl Acad Sci USA. 2011; 108: 3749-54.

30. Sulheim E, Iversen TG, Nakstad VT, Klinkenberg G, Sletta H, Schmid R, et al. Cytotoxicity of Poly(Alkyl Cyanoacrylate) Nanoparticles. Int J Mol Sci. 2017;

31. Villanueva C, Bazan F, Kim S, Demarchi M, Chaigneau L, Thiery-Vuillemin A, et al. Cabazitaxel A Novel Microtubule Inhibitor. Drugs. 2011; 71: 1251-8.

32. Cisternino S, Bourasset F, Archimbaud Y, Semiond D, Sanderink G, Scherrmann JM. Nonlinear accumulation in the brain of the new taxoid TXD258 following saturation of P-glycoprotein at the blood-brain barrier in mice and rats. Brit J Pharmacol. 2003; 138: 1367-75.

33. Vrignaud P, Semiond D, Benning V, Beys E, Bouchard H, Gupta S. Preclinical profile of cabazitaxel. Drug Des Dev Ther. 2014; 8: 1851-67.

34. Ghoochani A, Majernik GH, Sehm T, Wach S, Buchfelder M, Taubert H, et al. Cabazitaxel operates anti-metastatic and cytotoxic via apoptosis induction and stalls brain tumor angiogenesis. Oncotarget. 2016; 7: 38306-18.

35. Girard E, Ditzler S, Lee D, Richards A, Yagle K, Park J, et al. Efficacy of cabazitaxel in mouse models of pediatric brain tumors. Neuro-Oncology. 2015; 17: 107-15

36. Paller CJ, Antonarakis ES. Cabazitaxel: a novel second-line treatment for metastatic castration-resistant prostate cancer (vol 5, pg 117, 2011). Drug Des Dev Ther. 2011; 5: 183-

37. Bouchet BP, Galmarini CM. Cabazitaxel, a New Taxane with Favorable Properties. Drug Today. 2010; 46: 735-42.

38. Duran GE, Derdau V, Weitz D, Philippe N, Blankenstein J, Atzrodt J, et al. Cabazitaxel is more active than first-generation taxanes in ABCB1(+) cell lines due to its reduced affinity for P-glycoprotein. Cancer Chemoth Pharm. 2018; 81: 1095-103.

39. Cho EE, Drazic J, Ganguly M, Stefanovic B, Hynynen K. Two-photon fluorescence microscopy study of cerebrovascular dynamics in ultrasound-induced blood-brain barrier opening. J Cerebr Blood F Met. 2011; 31: $1852-62$

40. Kobus T, Zervantonakis IK, Zhang YZ, McDannold NJ. Growth inhibition in a brain metastasis model by antibody delivery using focused ultrasound-mediated blood-brain barrier disruption. J Controlled Release. 2016; 238: 281-8.

41. Poon C, McMahon D, Hynynen K. Noninvasive and targeted delivery of therapeutics to the brain using focused ultrasound. Neuropharmacology. 2017; 120: 20-37. 